\title{
BEST FILTERS FOR THE GENERAL FATOU BOUNDARY LIMIT THEOREM
}

\author{
JÜRGEN BLIEDTNER AND PETER A. LOEB
}

(Communicated by Albert Baernstein II)

\begin{abstract}
Given a suitable normalization, there is a "best" family of filters for which the Fatou Boundary Limit Theorem holds. The normalization assigns to each positive harmonic function a set of boundary points at which that function must vanish. Known limits, such as those provided by the Lebesgue Differentiation Theorem, are used to force consistency in this assignment. The zero sets, in turn, are used in constructing the coarsest filters which produce those limits almost everywhere. This procedure is formulated in terms of a general potential theoretic setting and a general reference measure. The result is new, however, even for harmonic functions on the unit disk.
\end{abstract}

\section{INTRODUCTION}

In [4], J. L. Doob showed that for positive harmonic functions on the unit disk, there is no "best", i.e., coarsest, filter for which a Fatou Boundary Limit Theorem holds when the filter is copied by rotation at all points of the unit circle. In this note we use the principal result from [3] to show that after a suitable normalization of the problem, there is a "best" family of filters for approaching the boundary. The normalization assigns to each positive harmonic function a set of boundary points at which that function must vanish. Known limits, such as those given by the Lebesgue Differentiation Theorem, guide in making this assignment. The zero sets are then used in constructing a filter of approach neighborhoods at each boundary point. These filters are the coarsest ones for which a Fatou Boundary Limit Theorem holds and the required zero limits are achieved.

The result we use from [3] is a necessary and sufficient condition for almost everywhere convergence; the corresponding filters are the smallest collections of large neighborhoods producing the desired limits. This suggests that sets from other filters, such as nontangential neighborhoods, work because they are subsets of the neighborhoods constructed here. Indeed, in retrospect, one finds

Received by the editors January 7, 1993 and, in revised form, May 10, 1993; presented to the society at the 1993 winter meeting in San Antonio.

1991 Mathematics Subject Classification. Primary 31A20, 31B25, 31C35; Secondary 30E25, $30 \mathrm{~F} 25$.

Key words and phrases. Boundary limits, Fatou Theorem, zero sets.

The second author's work was supported in part by a grant from the U.S. National Science Foundation. 
this containment at the heart of the short proof of the fine limit theorem given by the authors in [2].

Our principal result will be stated for very general, potential theoretic settings and general reference measures. The result is new, however, even for harmonic functions on the unit disk. One application for the disk, or any domain with the Martin boundary, is a construction of filters strictly coarser than those formed from minimal fine neighborhoods.

\section{FATOU-FILTER MAPPINGS}

Let $X$ be a compactifying boundary for a connected and locally connected, locally compact, but not compact Hausdorff space $W$. We assume that $W$ is the domain of definition for "harmonic functions" in a linear potential theoretic setting. Appropriate examples are a domain in a Euclidean space or a Riemann surface with ordinary harmonic functions, and even a domain in an axiomatic, potential theoretic setting (see [5], [1], and [7]). Let $\mathscr{B}$ denote the Borel subsets of $X$, and fix a finite reference measure $\sigma$ on $(X, \mathscr{B})$. Given any finite Borel measure $\nu$ and a set $E \in \mathscr{B}$, we write $\nu_{E}$ for the "restriction" of $\nu$ to $E$; i.e., $\nu_{E}(A)=\nu(A \cap E)$ for each $A \in \mathscr{B}$. We assume that $X$ supports an integral representation in the following sense:

(1) There is a convex cone $M$ of finite Borel measures on $X$, with $\sigma \in M$ and $\nu_{E} \in M$ when $\nu \in M$ and $E \in \mathscr{B}$.

(2) To each $\nu \in M$, there corresponds a harmonic function $h_{\nu}$ on $W$ so that the map $\nu \mapsto h_{\nu}$ is a positive affine map on $M$.

(3) The function $h_{\sigma}$ is strictly positive on $W$.

(4) If $\nu \in M$ and $h_{\sigma} \leq h_{\nu}$, then $\sigma \leq \nu$; i.e., for each $E \in \mathscr{B}, \sigma(E) \leq$ $\nu(E)$.

2.1. Example. A familiar example of such an integral representation is obtained by letting $X$ and $W$ be the unit circle $C$ and unit disk $D$, respectively, in the complex plane, letting $\sigma$ be normalized Lebesgue measure on $C$, setting $M$ equal to the set of finite Borel measures on $C$, and letting $h_{\nu}$ denote the Poisson integral of $\nu$ for each $\nu \in M$.

2.2. Example. Other integral representations can be found by letting $X$ be the Martin boundary [5], or the boundary constructed by the second author in [7], for the space of ordinary harmonic functions on a domain in Euclidean space or for a more general space of harmonic functions (see [5], [1], and [7]). Of course, one can always restrict $M$ to the set of all measures absolutely continuous with respect to the reference measure $\sigma$, or to measures generated by densities in $L^{p}$ for some $p$ with $1 \leq p \leq \infty$.

Given $\nu \in M$, we write $d \nu / d \sigma$ for the Radon-Nikodym derivative of the absolutely continuous part of $\nu$ with respect to $\sigma$.

2.3. Definition. By a Fatou-filter mapping we mean a function $\mathscr{G}: x \mapsto \mathscr{G}_{x}$ associating with each point $x \in X$ a filter base $\mathscr{G}_{x}$ of subsets of $W$ so that for each $\nu \in M$, the ratio $h_{\nu} / h_{\sigma}$ has limit $d \nu / d \sigma(x)$ along $\mathscr{G}_{x}$ (i.e., along the filter generated by $\mathscr{G}_{x}$ ) for $\sigma$-a.e. $x \in X$. That is,

$$
\lim _{\mathscr{G}_{x}} \frac{h_{\nu}}{h_{\sigma}}=\frac{d \nu}{d \sigma}(x) \quad \sigma \text {-a.e. }
$$


2.4. Remark. Given a filter mapping initially defined only $\sigma$-a.e. on $X$, we will assume that the mapping has been extended to the rest of $X$ with the filter containing only the set $W$.

2.5. Examples. For harmonic functions on the unit disk $D$ with the integral representation of Example 2.1, the radial approach, nontangential approach, and minimal fine approach all provide examples of Fatou-filter mappings. That is, these are all approaches to the boundary for which the Fatou Boundary Limit Theorem holds.

In what follows, we write $\left\{h_{\nu}<h_{\sigma}\right\}$ to denote the set $\left\{w \in W: h_{\nu}(w)<\right.$ $\left.h_{\sigma}(w)\right\}$. We will write $\sigma(A)=0$ if this is true for $A$ in the $\sigma$-completion of $\mathscr{B}$. We say that a Fatou-filter mapping $\mathscr{F}$ is coarser than a Fatou-filter mapping $\mathscr{G}$ if at each $x \in X$ the filter generated by $\mathscr{F}_{x}$ is contained in the filter generated by $\mathscr{G}_{x}$; we do not require the containment to be strict.

\section{ZERO SETS}

Our result is given in terms of a mapping $Z: \nu \mapsto Z_{\nu}$ from $M$ into the $\sigma$-completion of $\mathscr{B}$. We call $Z_{\nu}, \nu \in M$, the zero set for $\nu$, and we think of it as the set of required zeros of $d \nu / d \sigma$. We assume that the following conditions hold for all $\nu, \mu \in M$, all $E \in \mathscr{B}$, and all $\alpha>0$ in $\mathbb{R}$ :

(i) $Z_{\nu} \cap Z_{\mu} \subseteq Z_{\nu+\mu}$,

(ii) $Z_{\alpha \nu}=Z_{\nu}$,

(iii) $Z_{\nu}=\varnothing$ if $\sigma \leq \nu$,

(iv) $Z_{0}=X$,

(v) If $\nu(E)=0$, then $\sigma\left(E \backslash Z_{\nu}\right)=0$.

3.1. Examples. The above conditions will hold if each $Z_{\nu}$ is the set where a limit 0 is obtained when computing a representative for $d \nu / d \sigma$ using a differentiation basis and the Lebesgue Differentiation Theorem (see [3]). Here, for each nonzero $\nu \in M$, we associate a positive value at each point where the limit does not exist and at each point outside of the support of $\sigma$. We can also choose the zero sets by using a Fatou-filter mapping on $X$; for each $\nu \in M$ we take the lim sup of the ratio $h_{\nu} / h_{\sigma}$ along the filter at each $x \in X$ and put $x \in Z_{\nu}$ if the result is 0 . Since at least $W$ is in the filter at each point of $X$, condition (iv) is satisfied. These examples illustrate how, in practice, the selection of a zero set $Z_{\nu}$ takes into account the singular part of $\nu$.

Now, in terms of the choice of $M, \sigma$, and the mapping $Z$, we construct a best, i.e., a coarsest, Fatou-filter mapping. For each $x \in X$ we set

$$
\mathscr{F}_{x}:=\left\{\left\{h_{\nu}<h_{\sigma}\right\}: \nu \in M, x \in Z_{\nu}\right\} .
$$

3.2. Theorem. The mapping $\mathscr{F}: x \mapsto \mathscr{F}_{x}$ is a Fatou-filter mapping on $X$. It is the coarsest such mapping with the property that for each $\nu \in M$,

$$
Z_{\nu} \subseteq\left\{x \in X: \lim _{\mathscr{F}_{x}}\left(h_{\nu} / h_{\sigma}\right)=0\right\} .
$$

If $y \in X, \nu \in M$, and $h_{\nu} / h_{\sigma}$ has limit 0 along $\mathscr{F}_{y}$, then $\left\{h_{\nu}<h_{\sigma}\right\}$ is in the filter generated by $\mathscr{F}_{y}$.

Proof. First, fix $x \in X$. To show that $\mathscr{F}_{x}$ is a filter base, we note that by condition (iii), $\varnothing \notin \mathscr{F}_{x}$; if $\left\{h_{\nu}<h_{\sigma}\right\} \in \mathscr{F}_{x}$ and $\left\{h_{\mu}<h_{\sigma}\right\} \in \mathscr{F}_{x}$, then by 
condition (i), $\left\{h_{\nu+\mu}<h_{\sigma}\right\} \in \mathscr{F}_{x}$, and of course

$$
\left\{h_{\nu+\mu}<h_{\sigma}\right\} \subseteq\left\{h_{\nu}<h_{\sigma}\right\} \cap\left\{h_{\mu}<h_{\sigma}\right\} .
$$

To show that the mapping $\mathscr{F}$ is a Fatou-filter mapping on $X$, we fix a set $E \in \mathscr{B}$ and a measure $\nu \in M$ with $\nu(E)=0$. Since $\sigma\left(E \backslash Z_{\nu}\right)=0$, we have $\left\{h_{\nu}<h_{\sigma}\right\} \in \mathscr{F}_{x}$ for $\sigma$-a.e. $x \in E$. By Theorem 1.1 of [3] we are done. (Here, we are using the mappings $\nu \mapsto h_{\nu}(w), w \in W$, for the appropriate functionals on the elements $\nu \in M$; also see [2].) Fix $\nu \in M$. If $x \in Z_{\nu}$, then by condition (ii), for each natural number $n,\left\{n h_{\nu}<h_{\sigma}\right\} \in \mathscr{F}_{x}$, so the ratio $h_{\nu} / h_{\sigma}$ has limit 0 along $\mathscr{F}_{x}$. If $\mathscr{G}_{x}$ is also a filter of subsets of $W$ along which $h_{\nu} / h_{\sigma}$ has limit 0 , then $\left\{h_{\nu}<h_{\sigma}\right\} \in \mathscr{G}_{X}$. This shows that the mapping $x \mapsto \mathscr{F}_{X}$ is the coarsest Fatou-filter mapping on $X$ yielding the desired zeros. If $y \in X$ and $h_{\nu} / h_{\sigma}$ has limit 0 along $\mathscr{F}_{y}$, then there is a set $F \in \mathscr{F}_{y}$ on which $h_{\nu} / h_{\sigma}<1$, and so the set $\left\{h_{\nu}<h_{\sigma}\right\}$ is in the filter generated by $\mathscr{F} y$.

3.3. Remarks. The last statement of Theorem 3.2 shows that producing the filter map $x \mapsto \mathscr{F}_{x}$ is an idempotent operation. Once the sets of zeros $Z_{\nu}$, $\nu \in M$, are produced by the map in the sense of Example 3.1, they do not change if the process is repeated.

Suppose the sets of zeros $Z_{\nu}, \nu \in M$, are produced by taking limits with respect to a Fatou-filter map $x \mapsto \mathscr{G}_{x}$ such that for every potential $p$ on $W$, the ratio $p / h_{\sigma}$ has a limit 0 along $\mathscr{G}_{x}$ at $\sigma$-a.e. $x \in X$. Then for each potential $p$ on $W$ and every $x \in X$ where the limit of $p / h_{\sigma}$ is 0 , one can add the set $\left\{p<h_{\sigma}\right\}$ to the filter base $\mathscr{F}_{x}$ and generate a new filter base since $\mathscr{F}_{x}$ is a subset of the filter generated by $\mathscr{G}_{x}$ and $\left\{p<h_{\sigma}\right\}$ is an element of that filter. With this change, $\mathscr{F}$ is still a Fatou-filter mapping, and for each potential $p$, the ratio $p / h_{\sigma}$ will have limit 0 along $\mathscr{F}_{x}$ at $\sigma$-a.e. $x \in X$. In other words, the Ratio Fatou Limit Theorem given in terms of superharmonic functions holds for $\mathscr{F}$ (see [2]).

\section{COMPARISON WITH MINIMAL FINE AND ORDINARY NEIGHBORHOOD FILTERS}

Starting with solutions of the Laplace equation on a domain in $n$-space, $n \geq 2$, suppose that $X$ is the Martin boundary or the boundary produced in [7], that $M$ is the set of all finite Borel measures on the minimal points of $X$, and that $h_{\sigma} \equiv 1$ on $W$. Let $\mathscr{G}_{x}$ be the minimal fine filter at each minimal point $x \in X$ and $\mathscr{G}_{x}=\{W\}$ at each non-minimal point $x \in X$. Producing zero sets as in Example 3.1 with the Fatou-filter mapping $\mathscr{G}$ (see [2]) and applying Theorem 3.2 yields a filter at each minimal point strictly coarser, i.e., strictly contained, in the minimal fine filter. This follows from the fact that any countable dense set is thin at each minimal point and the sets $\left\{h_{\nu}<h_{\sigma}\right\}$, $\nu \in M$, are open subsets of $W$. The following general result is also applicable at each point of $X$ if $X$ contains at least two points and is regular for the Dirichlet problem.

In what follows, we write $\mathscr{N}_{x}$ for the collection of all open sets $U$ in the compact space $W \cup X$ with $x \in U$.

4.1. Theorem. Suppose in the setting of Theorem 3.2 that $h_{\sigma} \equiv 1$ on $W$. Fix $x \in X$, and assume that for each $U \in \mathscr{N}_{x}$ there is a measure $\nu \in M$ with $x \in Z_{\nu}$ and $\inf \left\{h_{\nu}(w): w \in W \backslash U\right\}>0$. Then the filter generated by $\mathscr{F}_{x}$ 
refines the ordinary neighborhood filter, i.e., it contains the trace on $W$ of each $U \in \mathscr{N}_{x}$.

Proof. Fix $U \in \mathscr{N}_{x}$ and a corresponding $\nu \in M$ given by the hypothesis. Then for some $\alpha>0$, the nonempty set $\left\{\alpha h_{\nu}<1\right\}=\left\{h_{\alpha \nu}<1\right\} \subset U$.

\section{BEST FILTERS AND DoOB's RESUlt}

Finally, we return to Example 2.1, i.e., harmonic functions on the unit disk $D$ with $\sigma$ equal to normalized Lebesgue measure on the boundary $C$. Suppose that $\nu$ is a finite measure on $C$ constructed from a sequence of point masses with the points converging to 1 but with 1 a point of symmetric density 0 for $\nu$. Then the radial and nontangential limit at 1 of $h_{\nu}$ is 0 . This is shown for the radial limit, in Theorem 3.1 of [6], and the result for the nontangential limit follows from Harnack's inequality using the argument at the end of the proof of Theorem 3.9 of [6]. (In general, the nontangential Fatou Boundary Limit Theorem for the disk can be proved using Theorem 1.1 of [3] and an argument, similar to what we have just given, which shows that $\lim h_{\mu}=0$ for $\sigma$-almost every point of a Borel set $E$ when $\mu$ is a measure with $\mu(E)=0$.)

Let $M_{c}$ consist of all measures with continuous densities on $C$. Define a filter mapping $\mathscr{G}$ on $C$ by setting $\mathscr{G}_{x}=\left\{U \cap D: U \in \mathscr{N}_{x}\right\}$ for each $x \in C$. Then $\mathscr{G}$ is a Fatou-filter mapping for the convex cone generated by $M_{c}$ and $\nu$, but $h_{\nu}$ does not have limit 0 along $\mathscr{G}_{1}$. Of course, by adding sets to $\mathscr{G}_{1}$ of the form $\left\{h_{\alpha \nu}<1\right\}, \alpha>0$, we will generate a filter along which $h_{\nu}$ does converge to 0 at 1 . With a similar modification of the filter $\mathscr{G}_{x}$ at each point $x=e^{i \theta}$ we can obtain a limit 0 at $x$ for the measure $\nu$ rotated by the angle $\theta$. These modifications are not needed, however, if we only want to obtain a limit 0 for $h_{\nu}$ at $\sigma$-a.e. point of $C$. This example demonstrates, in a simplified setting, the difference between Doob's result [4] and Theorem 3.2: There need not be a coarsest Fatou-filter map for all of $M$ if the zeros are not specified.

\section{REFERENCES}

1. J. Bliedtner and W. Hansen, Potential theory, Springer-Verlag, Berlin, 1986.

2. J. Bliedtner and P. A. Loeb, A measure-theoretic boundary limit theorem, Arch. Math. 43 (1984), 373-376.

3. $\ldots$ A reduction technique for limit theorems in analysis and probability theory, Ark. Mat. 30 (1992), 25-43.

4. J. L. Doob, Boundary approach filters for analytic functions, Ann. Inst. Fourier (Grenoble) 23 (1973), 187-213.

5. 1984.

6. L. L. Helms, Introduction to potential theory, Wiley, New York, 1969.

7. P. A. Loeb, A regular metrizable boundary for solutions of elliptic and parabolic differential equations, Math. Ann. 251 (1990), 43-50.

Fachbereich Mathematik, Universität Frankfurt, Robert-Mayer-Strasse 6-8, 60054, Frankfurt/M, FEDERAL REPUblic OF GERMANY

E-mail address: bliedtne@math.uni-frankfurt.de

Department of Mathematics, University of Illinois, 1409 West Green Street, Urbana, ILLINOIS 61801

E-mail address: loebomath.uiuc.edu 